\title{
(2) OPEN ACCESS \\ Long-term outcomes of ocular adnexal lesions in IgG4-related ophthalmic disease
}

\author{
Toshinobu Kubota (1), ${ }^{1}$ Masao Katayama, ${ }^{2}$ Reiko Nishimura, ${ }^{3}$ Suzuko Moritani ${ }^{4}$
}

- Additional material is published online only. To view, please visit the journal online (http://dx.doi.org/10.1136/ bjophthalmol-2018-313730).

'Department of Ophthalmology, National Hospital Organization Nagoya Medical Center, Nagoya, Japan

2Department of Rheumatology, National Hospital Organization Nagoya Medical Center, Nagoya, Japan

${ }^{3}$ Department of Pathology, National Hospital Organization Nagoya Medical Center, Nagoya Japan

${ }^{4}$ Clinical Laboratory Medicine and Division of Diagnostic Pathology, Shiga University Medical Science, Otsu, Japan

Correspondence to Dr Toshinobu Kubota, National Hospital Organization Nagoya Medical Center, Nagoya 4600001, Japan; ganiky9@gmail. com

Received 13 December 2018 Revised 29 May 2019 Accepted 6 June 2019 Published Online First 4 July 2019
ABSTRACT

Aims To determine the long-term outcomes of ocular adnexal lesions in immunoglobulin G4-related ophthalmic disease (IgG4-ROD).

Methods This retrospective, non-randomised exploratory study included 82 patients with ocular adnexal lesions. We evaluated the long-term outcomes in 71 patients during the median follow-up period of 30 months, who underwent either watchful waiting ( $n=20$; range 12-90 months) or systemic corticosteroid treatment, delivered according to consensus guidelines ( $n=51$; range $9-115$ months). We also analysed factors that might contribute to recalcitrance to treatment. Results of 82 patients, 40 (49\%) were male, and the median patient age was 60 years old. Twenty-one (26\%) patients with extraocular muscle (EOM) and/ or trigeminal nerve branch (CN V) enlargements had a significantly high frequency of multiple ocular adnexal lesions ( $p<0.0001$, Fisher's exact test). In addition, two patients developed EOM and/or CN V enlargements de novo over time. Twenty patients with solitary lacrimal gland enlargements preferred watchful waiting, due to mild symptoms. Of these, 18 (90\%) lesions remained dormant throughout a median follow-up of 27 months. Among 51 patients treated with corticosteroids, 31 (61\%) experienced relapses after treatment and required systemic low-dose maintenance treatment. A multivariate analysis indicated that EOM and/or CN V enlargements comprised a risk factor for relapse (HR 2.7; 95\% CI 1.1 to 6.7).

Conclusions This exploratory study showed that different types of ocular adnexal lesions in IgG4-ROD displayed distinct proliferative activities. Our results suggested that EOM and/or CN V enlargements might be secondary lesions that confer refractoriness to systemic corticosteroid treatment recommended by consensus guidelines.

\section{INTRODUCTION}

Immunoglobulin G4 (IgG4)-related disease is characterised by typical IgG4-positive lymphoplasmacytic infiltrative lesions and occasionally with elevated serum IgG4 levels. ${ }^{1-3}$ Ocular adnexal tissues, such as the lacrimal gland (LG), extraocular muscles (EOMs), trigeminal nerve branches (cranial nerve $(\mathrm{CN}) \mathrm{V}$ ) and orbital fat, are affected by systemic IgG4-related conditions. These effects are collectively called IgG4-related ophthalmic disease (IgG4-ROD). Systemic corticosteroid treatment was reported to be the first-line treatment according to an international consensus on IgG4-RD management. ${ }^{4}$ Several studies have reported serological risk factors related to relapse of ocular adnexal lesions after treatment. ${ }^{5}$ However, the long-term outcomes of ocular adnexal lesions in IgG4-ROD have not been well described. We hypothesised that there might be different proliferative activities, clinical behaviours and prognoses among different types of ocular adnexal lesions. Accordingly, it might be important to establish optimal management and treatments for each type of lesion. To verify this hypothesis, we retrospectively studied the long-term outcome of ocular adnexal lesions in IgG4-ROD, after either systemic corticosteroid treatment, based on consensus guidelines, or watchful waiting.

\section{PATIENTS AND METHODS \\ Patients and clinical data}

All patients provided informed written consent for the use of their data after the procedures were performed and the possible outcomes were explained to them.

This study was an exploratory retrospective cohort study. We performed histological and immunohistochemical examinations of biopsy specimens and obtained laboratory data for all patients diagnosed with definite or probable IgG4-ROD between 01 February 2005 and 31 August 2016. Eighty-two patients with definitive $(n=71)$ and probable $(n=11)$ IgG4-ROD were enrolled, based on the proposed criteria (box 1 ). ${ }^{7}$ Previous reports described 2, 15 and 30 of these patients, ${ }^{689}$ but the clinical follow-up data were updated for the present study.

A flowchart of patient selection and treatment groups is shown in figure 1. Rheumatologists administered corticosteroid and different regimens of other immunosuppressive treatments immediately prior to the treatment regimen for 6 patients, due to multiple systems and organs involvement. The remaining 76 patients provided informed consent for systemic corticosteroid treatment. When the corticosteroid treatment was refused, we proposed other alternatives, and the patients selected the treatment based on their preference. As a result, 54 patients underwent systemic corticosteroid treatment, based on the regimen, and 3 of these added 30 Gy radiotherapy to the treatment. Among the 54 patients that selected systemic corticosteroid treatment, 3 patients were lost to follow-up within 6 months of starting treatment. Another 2 patients of the 76 underwent other, alternative treatments; of the latter, 1 patient underwent only 30 Gy radiotherapy, and 1 patient selected local corticosteroid injections. ${ }^{10}$ Finally, 20 of the 76 patients had solitary LG enlargements, and preferred watchful 
Box 1 Diagnostic criteria for immunoglobulin $\mathrm{G} 4$ (lgG4)related ophthalmic disease, $2014^{7}$

1. Imaging studies show enlargement of lacrimal gland, trigeminal nerve or extraocular muscle as well as masses, enlargement or hypertrophic lesions in various ophthalmic tissues.

2. Histopathological examination shows marked lymphocyte and plasmacyte infiltration, and sometimes fibrosis. A germinal centre is frequently observed. IgG4+ plasmacytes are found and satisfy the following criteria: ratio of $\lg \mathrm{G} 4+$ cells to lg $\mathrm{g}+$ cells of $40 \%$ or above, or more than $50 \mathrm{lgG} 4+$ cells per high-power field $(\times 400)$.

3. Blood test shows elevated serum lgG4 ( $\geq 135 \mathrm{mg} / \mathrm{dL}$ ).

Diagnosis is classified as 'definitive' when 1, 2 and 3 are satisfied; 'probable' when 1 and 2 are satisfied; and 'possible' when 1 and 3 are satisfied.

waiting, due to mild signs and symptoms. Therefore, we analysed the long-term outcomes of ocular adnexal lesions in 71 patients, after either systemic corticosteroid treatment $(n=51)$ or watchful waiting $(n=20)$.

\section{Anatomic locations of ocular adnexa}

We determined the anatomic locations of ocular adnexal lesions in all patients by reviewing CT scans or MRIs. We recorded each patient's laterality. Patients were divided into three groups, based on the anatomic location of the lesions, as follows: LG group: LG enlargement; EOM/CN V group: EOM and/or CN V enlargements; and other lesions group: orbital fat, perioptic and periocular infiltrations.

\section{Laboratory data}

Peripheral blood was collected from all patients at the time of diagnosis and at each follow-up visit. Haematological examinations were performed with an emphasis on the serum IgG4 level. Blood eosinophilia was diagnosed when the number of eosinophils was $\geq 600$ cells $/ \mathrm{mm}^{3} .{ }^{11}$ Serum levels of rheumatoid factor (latex turbidimetric immunoassay, normal $<10 \mathrm{IU}$ ) were determined at the time of diagnosis in all patients. When the

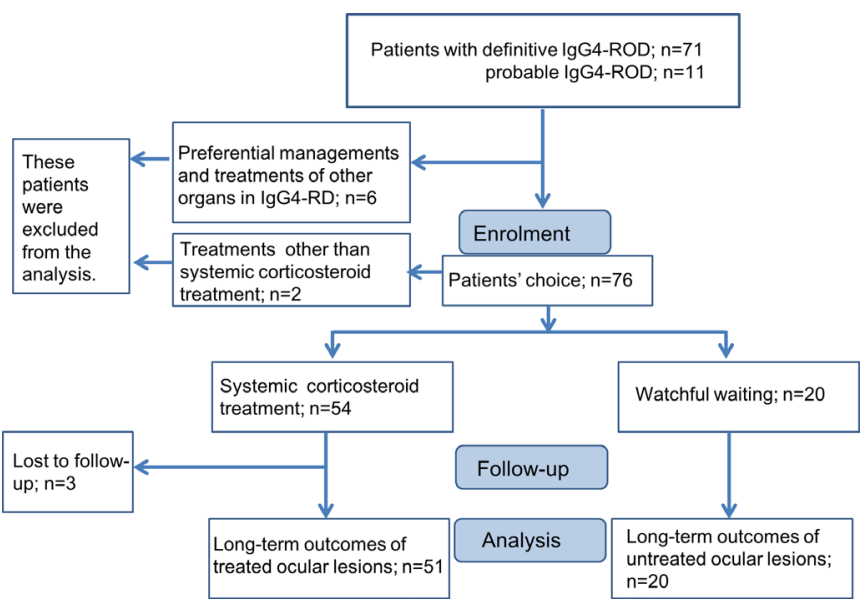

Figure 1 Flowchart of patient selection and treatment groups. IgG4-RD, immunoglobulin G4-related disease; IgG4-ROD, IgG4-related ophthalmic disease. rheumatoid factor level was $\geq 30 \mathrm{IU}$, the patient was classified as positive for rheumatoid factor.

\section{Systemic evaluations}

To determine systemic involvement, 49 (69\%) patients were screened during the initial examinations as follows: whole-body CT, 4 patients; gallium-67 imaging, 36 patients; and ${ }^{18} \mathrm{~F}$-fluorodeoxyglucose positron emission tomography, 9 patients. The remaining 22 patients preferred immediate treatment or refused to undergo screening for systemic involvement.

\section{Treatments and outcomes}

Patients received systemic corticosteroid treatments, according to consensus guidelines. ${ }^{4}$ However, it remains controversial whether low-dose corticosteroid treatment should be maintained for years. ${ }^{4}$ Prednisolone was given at $30 \mathrm{mg} /$ day or 0.6 $\mathrm{mg} / \mathrm{kg} /$ day for 1 or 2 weeks, and this dose was reduced by $5 \mathrm{mg} /$ day at 2-week intervals. After discontinuing corticosteroids, each patient's condition was followed, and occurrences of regression and recurrence of ocular adnexal lesions in IgG4-ROD were determined radiologically. To evaluate the response to treatment and the incidence of orbital lesion relapses, we compared axial and coronal CTs and MRIs, according to previously described methods. ${ }^{6}$ At the time of relapse, systemic corticosteroid treatment was restarted in the same manner each time, or it was restarted in the same manner, but maintained at a low dose (prednisolone; range $5-10 \mathrm{mg} /$ day) with very slow tapering over years.

\section{Statistical analyses}

We performed univariate analyses, followed by the log-rank test, and multivariate analyses, followed by the Cox hazard model to determine risk factors for relapse after discontinuation of systemic corticosteroid treatment. To explore patient characteristics, we analysed patient demographic data, anatomic locations of lesions, laboratory data and combinations of these factors with the $\chi^{2}$ test and Fisher's exact test.

All statistical analyses were performed with the statistical package, SPSS for Windows V.12.0, and the statistical software, EZR. ${ }^{12} \mathrm{~A} p$ value $<0.05$ was considered statistically significant.

\section{RESULTS \\ Clinical data, anatomic locations of lesions and laboratory data}

Forty (49\%) patients were male. The median patient age was 60 years old (range 25-82 years). Fifty-seven patients (70\%) had bilateral lesions. Sixty-three patients $(77 \%)$ had a single type of lesion; of these, 49 had LG enlargements; 2 had EOM/CN $\mathrm{V}$ enlargements; and 12 had other lesions. The remaining 19 patients $(23 \%)$ had multiple types of lesions; of these, 10 patients had LG enlargements and EOM/CN V enlargements; 4 patients had $\mathrm{EOM} / \mathrm{CN} \mathrm{V}$ enlargements and other lesions; and 5 patients had all three types of lesions. Twenty-one (26\%) patients with $\mathrm{EOM} / \mathrm{CN} \mathrm{V}$ enlargements had a significantly high frequency of multiple ocular adnexal lesions ( $\mathrm{p}<0.0001$, Fisher's exact test).

Fifteen (18\%) patients had EOM enlargements. Among these, the lateral rectus muscle was the most common lesion $(n=14 / 15$ patients; 93\%), followed by the inferior rectus muscle $(n=9$; $60 \%)$, superior rectus muscle $(\mathrm{n}=8 ; 53 \%)$, medial rectus muscle $(\mathrm{n}=5 ; 33 \%)$ and superior oblique muscle $(\mathrm{n}=2 ; 13 \%)$. Nine (11\%) patients had CN V enlargements. Among these, six (67\%) patients had enlargements of the solitary inferior orbital nerves; one $(11 \%)$ had an enlargement of the solitary superior orbital 
Table 1 Univariate and multivariate analysis results show risk factors for IgG4-related ophthalmic disease relapse after systemic corticosteroid treatment

\begin{tabular}{|c|c|c|c|c|c|}
\hline \multirow[b]{2}{*}{ Factor } & \multirow{2}{*}{$\begin{array}{l}\text { Univariate analysis } \\
\text { (Log-rank test) } \\
\text { P value }\end{array}$} & \multicolumn{2}{|c|}{$\begin{array}{l}\text { Multivariate analysis A } \\
\text { (Cox hazard model) }\end{array}$} & \multicolumn{2}{|c|}{$\begin{array}{l}\text { Multivariate analysis B } \\
\text { (Cox hazard model) }\end{array}$} \\
\hline & & HR $(95 \% \mathrm{Cl})$ & $P$ value & HR $(95 \% \mathrm{Cl})$ & $P$ value \\
\hline Age (>60 years) & 0.6 & - & & - & \\
\hline Sex (male) & $0.04^{*}$ & - & & $1.4(0.6$ to 3.3$)$ & 0.4 \\
\hline Complicationst & 0.8 & - & & - & \\
\hline \multicolumn{6}{|l|}{ Anatomic locations } \\
\hline Laterality (bilateral) & 0.5 & - & & - & \\
\hline Lacrimal glands & 0.7 & - & & - & \\
\hline EOMs and CN V & $0.0002^{*}$ & $2.7(1.1$ to 6.7$)$ & $0.03^{*}$ & $2.5(1.0$ to 6.3$)$ & $0.04^{*}$ \\
\hline Other ocular adnexal lesions & 0.3 & - & & - & \\
\hline Systemic involvement & 0.5 & - & & - & \\
\hline \multicolumn{6}{|l|}{ Histology } \\
\hline $\operatorname{lgG} 4: \operatorname{lgG}$ ratio $(>0.6)$ & 0.8 & - & & - & \\
\hline \multicolumn{6}{|l|}{ Serological data } \\
\hline Serum lgG4 level (>900 mg/dL) & 0.3 & - & & - & \\
\hline Serum lgG4 level (>400 mg/dL) & $0.01^{*}$ & $1.4(0.6$ to 3.6$)$ & 0.5 & $1.4(0.5$ to 3.5$)$ & 0.5 \\
\hline Blood eosinophilia (yes) & $0.02^{*}$ & - & & - & \\
\hline Rheumatoid factor (positive) & $0.04^{*}$ & $1.1(0.5$ to 2.7$)$ & 0.8 & - & \\
\hline
\end{tabular}

${ }^{*}$ Statistically significant.

†Complications of IgG4-related ophthalmic disease include asthma, rhinitis refractory to conventional medications, atopic dermatitis and hyposmia.

CN V, trigeminal nerve; EOMs, extraocular muscles; IgG4, immunoglobulin G4.

nerves; and two (22\%) had enlargements of both the inferior and superior nerves. Among the 82 patients enrolled, 5 (6\%) patients had both the EOM and CN V enlargements.

Laboratory data showed that the median serum IgG4 level was $448 \mathrm{mg} / \mathrm{dL}$ (range 23-4501 mg/dL; normal 5-105 mg/dL). Eighteen $(22 \%)$ patients had high IgG4 serum levels $(\geq 900 \mathrm{mg} / \mathrm{dL})$, $15(18 \%)$ patients had blood eosinophilia and $16(20 \%)$ patients were positive for rheumatoid factor.

Of 49 patients, who were evaluated for systemic involvement, 32 patients $(65 \%)$ had systemic involvement at the time of diagnosis; 27 (55\%) had lesions in the salivary glands, 7 $(14 \%)$ had lesions in the maxillary or ethmoid sinuses and 17 (35\%) had lesions in the lymph nodes. Seven (14\%) patients had other systemic involvement at the time of diagnosis, as follows: kidneys, three; pancreas, three; retroperitoneum and periaortic region, two; and unusual mammary (biopsy proven) and sublingual lesions, one.

\section{Treatments and outcomes}

We analysed data for 51 patients that underwent systemic corticosteroid treatment according to the regimen. After the treatment, the patients underwent both the clinical and radiological examinations during the follow-up period. Among these, 31 (61\%) patients exhibited relapses during a median follow-up of 41 months (range 17-115 months). We performed univariate and multivariate analyses of potential risk factors related to relapse after systemic corticosteroid treatment (table 1). Univariate analysis with the log-rank test showed that male sex $(p=0.04), E O M / C N$ V enlargements $(p=0.0002)$, elevated serum IgG4 levels $(>400 \mathrm{mg} / \mathrm{dL}, \mathrm{p}=0.01)$, blood eosinophilia $(p=0.02)$ and positivity for rheumatoid factor $(p=0.04)$ were significant risk factors for relapse after systemic corticosteroid treatment. The multivariate analysis with the Cox hazard model showed that EOM/CN V enlargement was the only significant factor in both a factor A model (EOM/CN V enlargement, elevated serum IgG4 level and positivity for rheumatoid factor;
HR 2.7; 95\% CI 1.1 to 6.7) and a factor B model (EOM/CN V enlargement, elevated serum IgG4 level and sex; HR 2.5; 95\% CI 1.0 to 6.3 ). All patients that had a relapse required repeated systemic corticosteroid treatments. Treatments were repeated for a maximum of three rounds in patients that did not receive maintenance low-dose systemic corticosteroid treatment. Among patients that received maintenance treatment, three patients relapsed (figure 2), and one patient developed EOM and CN V enlargements de novo (figure 3) during the maintenance treatment of prednisolone at $5 \mathrm{mg} /$ day or less. The ocular adnexal

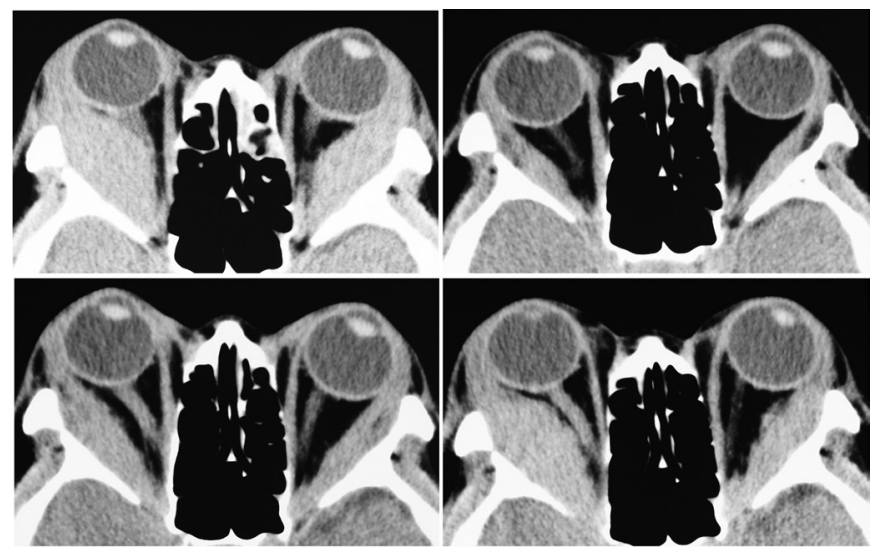

Figure $2 \mathrm{CT}$ images show rigorous proliferative lesion activities. Top left: Rapid relapse of bilateral lateral rectus muscles after the first systemic corticosteroid treatment. Top right: Partial regression of bilateral lesions after the second systemic corticosteroid treatment with $15 \mathrm{mg} /$ day prednisolone. Bottom left: Partial relapse of bilateral lateral rectus muscles at the time of a second systemic corticosteroid treatment with $5 \mathrm{mg} /$ day prednisolone. Bottom right: Further relapse of bilateral lateral rectus muscles at 21 months during maintenance low-dose systemic corticosteroid treatment with $5 \mathrm{mg} /$ day prednisolone. 


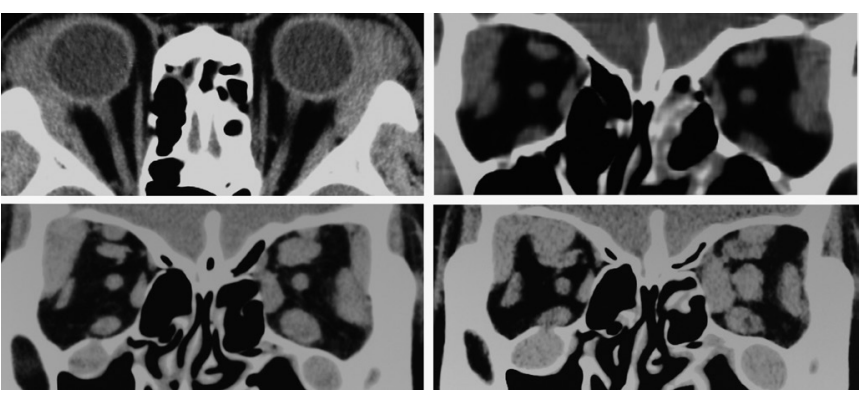

Figure 3 Enlargement of ocular adnexal lesions over time. Top, left and right: Enlargement of bilateral lacrimal glands at the initial examination. Bottom left: Enlargements of bilateral lacrimal glands, superior and inferior orbital nerves and all rectus muscles of the left eye, 12 months later, after discontinuing the first systemic corticosteroid treatment. Bottom right: Enlargements of bilateral superior and inferior orbital nerves, right orbital lacrimal gland, all rectus muscles of the left eye and optic nerve during maintenance prednisolone $(5 \mathrm{mg} /$ day or less), 23 months later, after a second systemic corticosteroid treatment.

lesions became dormant when low-dose maintenance treatment was increased to prednisolone at $10 \mathrm{mg} /$ day. In contrast, in 21 patients that did not relapse after the primary treatment in the same regimen, ocular adnexal lesions had remained dormant throughout the median follow-up period of 24 months (range 9-86 months).

Of the 20 patients that preferred watchful waiting, all had solitary, unilateral $(n=6)$ or bilateral $(n=14)$ LG enlargements. However, two patients (10\%) developed another lesion. Of these, one patient developed a unilateral infraorbital enlargement at 47 months after the initial visit; and one patient developed a contralateral periocular enlargement at 44 months after the initial visit, but without increasing the LG enlargement. In the remaining $18(90 \%)$ patients, the lesions remained dormant or regressed spontaneously (online supplementary file) during the median follow-up period of 27 months (range 12-90 months).

Patients with EOM/CN V enlargements had a significantly greater incidence of blood eosinophilia compared with the other groups ( $p=0.00006, \chi^{2}$ test) and elevated serum levels of IgG4 $(\geq 900 \mathrm{mg} / \mathrm{dL}$, normal $5-105 \mathrm{mg} / \mathrm{dL}, \mathrm{p}=0.01$, Fisher's exact test).

\section{DISCUSSION}

Patients with different types of IgG4-ROD had distinct clinical courses during the follow-up period. In patients with EOM/CN $\mathrm{V}$ enlargements, maintenance low-dose systemic corticosteroid treatment was frequently required to prevent enlargement of the lesions. In contrast, in patients with solitary LG enlargements, lesions often remained dormant without any treatment. Our statistical analyses indicated that EOM/CN V enlargements were a strong risk factor for recalcitrance to systemic corticosteroid treatment. Thus, the different affected ocular adnexal lesions might have different proliferative activities, clinical behaviours and prognoses.

Patients with EOM/CN V enlargements might be refractory to systemic corticosteroid treatment, delivered according to consensus guidelines. In addition, $\mathrm{EOM} / \mathrm{CN} \mathrm{V}$ enlargements might be secondary ocular adnexal IgG4-related lesions, based on the following three points. First, in two patients of our study, EOM/CN V enlargements developed independently 12 and 47 months later, after an ipsilateral solitary LG enlargement. Thus, in those cases, EOM/CN V enlargements did not appear to be infiltrations; instead, they seemed to be secondary de novo lesions. Second, previously published data and the results of our study revealed that patients with $\mathrm{EOM} / \mathrm{CN} \mathrm{V}$ enlargements had a significantly greater incidence of ipsilateral ocular adnexal involvement, compared with patients with other types of lesion ${ }^{13-16}$; this finding could indirectly support the notion that EOM/CN V enlargements are secondary lesions. Finally, EOM/CN V enlargements might be unique lesions, because skeletal muscles and the central nervous system are seldom affected in systemic IgG4-RD. ${ }^{317} \mathrm{EOM} / \mathrm{CN}$ V enlargements were affected in 19\%-44\% of patients with IgG4-ROD in previous studies and in the present study. ${ }^{13-15}{ }^{18}$ In other words, EOM/CN V enlargements might represent a progressive condition of IgG4-ROD.

Previous studies showed that serum IgG4 levels, the presence of rheumatoid factor and male sex were risk factors for IgG4-ROD relapse after systemic corticosteroid treatment. ${ }^{5619}$ Our univariate analysis showed similar results. However, our multivariate analysis of potential risk factors for relapse did not include those factors; instead, our analysis showed that EOM/ $\mathrm{CN} \mathrm{V}$ enlargement was a strong risk factor. Interestingly, our clinical data also indicated that $\mathrm{EOM} / \mathrm{CN} \mathrm{V}$ enlargements were significantly associated with quite elevated serum IgG4 levels and blood eosinophilia. One explanation for this association might be that systemic immune activation, which is linked to elevated serum IgG4 levels and blood eosinophilia, might have a worse ocular condition.

This study had several limitations. First, the study design was retrospective. Thus, the results should be considered exploratory. Second, we combined EOM and CN V enlargements into a single category. This was necessary, because we could not determine clinical differences between solitary EOM and CN V enlargements. Third, of 21 patients with solitary LG enlargements that received systemic corticosteroid treatments, 4 (19\%) recurred after systemic corticosteroid treatment. We could not determine potential risk factors for these patients, although two of these patients were positive for rheumatoid factor. Fourth, rituximab is promising for the treatment of steroid-resistant cases. ${ }^{13}$ However, we did not use rituximab because it has not been approved in Japan for treating inflammatory diseases. Finally, we did not analyse the durations of ocular symptoms, because, often, patients did not remember them well. This lack of recall was probably due to the chronic and mild nature of the symptoms. Indeed, some patients with solitary LG enlargement demonstrated an indolent clinical course without any treatments for a prolonged period. In contrast, other patients with EOM/ $\mathrm{CN}$ V enlargement demonstrated rapid proliferative activities, such as that shown in figure 2 . In addition, we found a positive correlation between EOM/CNV enlargement and blood eosinophilia/elevated serum level of $\operatorname{IgG} 4$, and observed that several patients had EOM/CNV enlargement, following LG enlargement, such as that shown in figure 3 . Therefore, we speculate that both the degree of systemic immunologic imbalances and duration of IgG4-ROD might determine the progression of the disease.

In conclusion, our results suggested that different ocular adnexal lesions in IgG4-ROD might have different proliferative activities, clinical behaviours and prognoses. On the basis of our exploratory findings, we hypothesised that EOM/CN V enlargements might be secondary lesions. Moreover, patients with $\mathrm{EOM} / \mathrm{CN} \mathrm{V}$ enlargements were frequently refractory to systemic corticosteroid treatment, delivered according to consensus guidelines. Furthermore, patients with EOM/CN $\mathrm{V}$ enlargements appeared to be associated with quite elevated 
serum IgG4 levels and blood eosinophilia. Future studies are needed to provide additional evidence for these findings.

Correction notice This paper has been amended since it was published Online First. Dr Katayama's affiliation has been updated.

Contributors Design of the study (TK); conduct of the study (TK, MK, RN and SM); analysis and interpretation (TK, MK, RN and SM); and literature search (TK).

Funding The authors have not declared a specific grant for this research from any funding agency in the public, commercial or not-for-profit sectors.

Competing interests None declared.

Patient consent for publication Not required.

Ethics approval The Ethics Committee at Nagoya Medical Center, Nagoya, Japan approved this retrospective review of patient data. The procedures used in this study conformed to the tenets of the Declaration of Helsinki.

Provenance and peer review Not commissioned; externally peer reviewed.

Data availability statement All data relevant to the study are included in the article or uploaded as supplementary information.

Open access This is an open access article distributed in accordance with the Creative Commons Attribution Non Commercial (CC BY-NC 4.0) license, which permits others to distribute, remix, adapt, build upon this work non-commercially, and license their derivative works on different terms, provided the original work is properly cited, appropriate credit is given, any changes made indicated, and the use is non-commercial. See: http://creativecommons.org/licenses/by-nc/4.0/.

ORCID iD

Toshinobu Kubota http://orcid.org/0000-0003-0653-0267

\section{REFERENCES}

1 Deshpande V, Zen Y, Chan JK, et al. Consensus statement on the pathology of IgG4related disease. Mod Pathol 2012;25:1181-92.

2 Carruthers MN, Khosroshahi A, Augustin T, et al. The diagnostic utility of serum IgG4 concentrations in IgG4-related disease. Ann Rheum Dis 2015;74:14-18.

3 Stone JH, Zen Y, Deshpande V. IgG4-related disease. N Engl J Med 2012;366:539-51.
4 Khosroshahi A, Wallace ZS, Crowe JL, et al. International consensus guidance statement on the management and treatment of IgG4-related disease. Arthritis Rheumatol 2015;67:1688-99.

5 Hong JW, Kang S, Song MK, et al. Clinicoserological factors associated with response to steroid treatment and recurrence in patients with IgG4-related ophthalmic disease. Br J Ophthalmol 2018;102:1591-5.

6 Kubota T, Katayama M, Moritani S, et al. Serologic factors in early relapse of IgG4-related orbital inflammation after steroid treatment. Am J Ophthalmol 2013:155:373-9.

7 Goto H, Takahira M, Takahira M, et al. Diagnostic criteria for IgG4-related ophthalmic disease. Jpn J Ophthalmol 2015;59:1-7.

8 Kubota T, Moritani S. Orbital IgG4-related disease: clinical features and diagnosis. ISRN Rheumatol 2012;2012(1, supplement 64):1-5.

9 Kubota T, Moritani S, Katayama M, et al. Ocular adnexal IgG4-related lymphoplasmacytic infiltrative disorder. Arch Ophthalmol 2010;128:577-84.

10 Kubota T, Moritani S, Sakuma M. Tarsal IgG4-related disease. JAMA Ophthalmol 2015;133:e143272.

11 Tefferi A. Blood eosinophilia: a new paradigm in disease classification, diagnosis, and treatment. Mayo Clin Proc 2005:80:75-83.

12 Kanda Y. Investigation of the freely available easy-to-use software 'EZR' for medical statistics. Bone Marrow Transplant 2013;48:452-8.

13 Wallace ZS, Deshpande V, Stone JH. Ophthalmic manifestations of IgG4-related disease: single-center experience and literature review. Semin Arthritis Rheum 2014;43:806-17.

14 Ebbo M, Patient M, Grados A, et al. Ophthalmic manifestations in IgG4-related disease: clinical presentation and response to treatment in a French case-series. Medicine 2017;96:e6205.

15 Plaza JA, Garrity JA, Dogan A, et al. Orbital inflammation with lgG4-positive plasma cells: manifestation of IgG4 systemic disease. Arch Ophthalmol 2011;129:421-8.

16 Tiegs-Heiden CA, Eckel LJ, Hunt CH, et al. Immunoglobulin G4-related disease of the orbit: imaging features in 27 patients. AJNR Am I Neuroradiol 2014;35:1393-7.

17 Wolfson AR, Hamilos DL. Recent advances in understanding and managing lgG4related disease. F1000Res 2017:6.

18 Sogabe Y, Ohshima K-ichi, Azumi A, et al. Location and frequency of lesions in patients with IgG4-related ophthalmic diseases. Graefes Arch Clin Exp Ophthalmol 2014;252:531-8

19 Yamamoto M, Nojima M, Takahashi $\mathrm{H}$, et al. Identification of relapse predictors in lgG4-related disease using multivariate analysis of clinical data at the first visit and initial treatment. Rheumatology 2015:54:45-9. 